\title{
Combining ECT and clozapine in the treatment of clozapine-refractory schizophrenia and schizoaffective disorder - a pilot study
}

L Koen, MB ChB, MMed (Psych)

$C$ E van den Berg, $M B$ ChB

D J H Niehaus, MB ChB, MMed (Psych), FCPsych, DMed Ngaphakathi Workgroup, Department of Psychiatry, Stellenbosch University and Stikland Hospital, W Cape

Objective. Clozapine is the current gold standard treatment for severe treatment-refractory schizophrenia, but even so 40 $70 \%$ of these patients will continue to experience disabling symptoms when treated with clozapine monotherapy. Current clinical practice at Stikland Hospital holds that known clozapine-refractory schizophrenia patients who relapse due to non-compliance are treated with an initial combination of clozapine and ECT (if able to consent) when readmitted. The purpose of this study was to evaluate the validity of this practice.

Methods. Patients were divided into an ECT (EG) and non-ECT (CG) group. Clozapine was started and ECT administered as per protocol. Demographic data, psychiatric and medication history and data concerning adverse events were collected. Positive and Negative Symptom Scale (PANSS) scores were done at baseline and at days 21 and 42 .

Results. At discharge, although numerically the average increase in clozapine dose was lower and the reduction in length of stay was greater in the EG, none of the variables measured were statistically significantly different between groups. More concomitants were also used in the EG.

Conclusions. This pilot study represents the first controlled trial of ECT-clozapine bitherapy in a population with clozapinerefractory schizophrenia and schizoaffective disorder reported in the literature. The validity of our choice of current clinical practice in this population was not supported by our results. However, the study did provide us with preliminary evidence for the safety and efficacy of this combination. It would therefore be reasonable to continue to use this strategy in selected cases, at least until other clozapine-refractory treatment strategies become more available in our setting.
Schizophrenia is considered to be among the most severe, debilitating and persistent of all psychiatric disorders. Although the adult prevalence is approximately $1 \%$, studies have indicated that sufferers constitute close to $10 \%$ of the permanently disabled population.' As such, schizophrenia is a major public health problem and the negative economic consequences underscore the urgent need to develop strategies to improve treatment effectiveness. ${ }^{2}$

Despite great advances in the understanding and management of the disease, a significant portion of individuals with schizophrenia do not respond well to drug treatment. ${ }^{3}$ Management of these treatmentrefractory patients is particularly challenging as they often require longer periods of hospitalisation, with their care ultimately amounting to a disproportionately high share of the total cost of schizophrenia.' Although the advent of the second-generation antipsychotic drugs has been regarded as a breakthrough owing to improved side-effect profiles and patient satisfaction, claims of their superior efficacy in treatmentrefractory patients still remain unproven. ${ }^{4}$ To date, in terms of evidence base, clozapine remains the undisputed treatment of choice for patients with severe treatment-refractory schizophrenia. ${ }^{5.7}$

Unfortunately, even though clozapine remains the gold standard, recent data show that $40-70 \%$ of sufferers from treatmentrefractory schizophrenia treated with clozapine monotherapy, of adequate dosage and duration will continue to experience cognitive deficits and disabling symptoms, i.e. positive, negative or residual symptoms. ${ }^{46,8}$ Several factors have been implicated in patient failure to respond to clozapine monotherapy, including patient non-compliance and intolerable and/or serious side-effects such as agranulocytosis, seizures, sedation, hypersalivation and metabolic issues. ${ }^{3,9}$ However, for patients in whom these can be managed and who show at least partial response to monotherapy, effective clozapine augmentation treatment strategies need to be developed. ${ }^{4}$

Numerous different clozapine augmentation strategies have been described in the literature, but there is currently no actual evidence supporting one strategy over another, with research consisting mostly of case reports and small open-label, unreplicated 
studies. ${ }^{6,10-15}$ Strategies include adjunctive antipsychotic medications, mood stabilisers and novel anticonvulsants, antidepressants such as selective serotonin re-uptake inhibitors and others, glycinergic agents, dopamine agonists, mazindol, omega-3 fatty acids and electroconvulsive therapy (ECT).

The combined use of clozapine and ECT was first proposed in 1990 by Fink and reported on in 1991.9,15,16 A recent literature review on ECT-clozapine bitherapy in patients with treatmentrefractory schizophrenia previously treated with clozapine monotherapy revealed $72 \%$ of reported cases to have had a beneficial response on the combination treatment. ${ }^{17}$ To date, the largest single study reported on in the literature is an open-label study evaluating ECT-clozapine bitherapy in 11 clozapine nonresponders, with 8 meeting remission criteria after treatment. ${ }^{18}$

Safety issues could be regarded as one potential concern when considering the use of ECT-clozapine bitherapy. It is known that clozapine use may lead to an increased risk of seizures or even prolonged seizures due to the threshold-lowering effects of clozapine. ${ }^{19,20}$ Both ECT and clozapine may also cause cardiac arrhythmias, and memory effects have been described. 21-23 However, a recent review article on somatic augmentation strategies in clozapine-refractoriness by Tranulis et al. found that only seven incidences of significant side-effects in ECTclozapine bitherapy had been described, with no fatalities. ${ }^{24}$ They concluded ECT to be a safe and tolerable clozapine augmentation strategy.

In conclusion, although the body of evidence in the literature for the use of ECT-clozapine bitherapy in clozapine-refractory schizophrenia is still far from permitting comprehensive evidencebased conclusions, there seems to be some support with regard to both the efficacy and the safety of the combination. Owing to the rate of clozapine-refractory admissions seen at Stikland Hospital each year, clinicians have had to develop management guidelines to ensure a more structured approach to these clients. A decision was therefore made to use ECT as a first-line add-on strategy based on ECT accessibility in comparison with the limited or nonexistent availability of most of the other strategies described in the literature. Current clinical practice therefore dictates that known clozapine-refractory patients who relapse due to non-compliance are treated with the ECT-clozapine bitherapy when readmitted, if consent for ECT can be obtained. For patients who do not give consent for ECT, treatment with clozapine is restarted and once a maximum tolerable dose or $400 \mathrm{mg}$ is reached and they still have not shown an adequate response, add-on medication is initiated. This strategy was implemented at the same time as the naturalistic active control study.

\section{Methods}

\section{Subjects}

Ten known clozapine-refractory patients with a diagnosis of schizophrenia or schizoaffective disorder were recruited from the acute wards of Stikland Psychiatric Hospital, Western Cape. In order to be included patients had to meet the following criteria: (i) admission for relapse due to non-compliance ( $\geq 1$ month); (ii) DSM-IV-TR diagnosis of schizophrenia or schizoaffective disorder; (iii) known to be clozapine-refractory (see criteria below); (iv) the decision to restart clozapine and augment with ECT made independently by the patient's treating clinician; and (iv) age 18 - 60 (extremes included). Patients were excluded if (i) they had another Axis I diagnosis (excluding substance abuse); and (ii) if they had a significant unstable medical condition.

Patients were regarded as clozapine-refractory if they met the following criteria: (i) previously treated with clozapine because of being regarded as treatment resistant (continued psychosis despite adequate trials of at least two antipsychotics of two different groups with the dosage equivalent of $10 \mathrm{mg}$ of haloperidol (or maximum tolerable dosell; and (ii) despite being treated with clozapine at a dosage of at least $400 \mathrm{mg} /$ day, or the maximum tolerated dose, for at least 6 weeks, the patient had previously exhibited continued psychosis. (Continued psychosis was defined as clinical symptomatology that rated a score of at least 4 on one item of the positive scale of the Positive and Negative Symptom Scale (PANSS). ${ }^{25}$ )

\section{Procedures}

All patients who met the inclusion criteria were screened by the primary investigator after ECT had been discussed with them by their treating clinician. The patients who consented to the study were divided into two groups /depending on whether they gave consent for ECT or not). The treating clinician obtained consent for ECT and performed all additional investigations required for ECT as well as the necessary haematological monitoring prior to starting clozapine, independently of the study team.

Clozapine was started by the treating clinician las per normal treatment protocoll) at $25 \mathrm{mg} /$ day and the dose was titrated up every 2 days by $25 \mathrm{mg}$ until a dose of $150 \mathrm{mg}$ was reached. Thereafter the dose was titrated up by 50 mg every 2 days until $250 \mathrm{mg}$ was reached, whereafter titration of the dose continued according to ward protocol $150 \mathrm{mg} /$ week until response or maximum tolerated dose was reached). 
ECT was started by the treating clinician between days 8 and 10 after admission and administered as per the usual Stikland Hospital protocol on alternate days for three sessions per week for six sessions in total. Concomitant medications were only considered (at the sole discretion of the ward clinicians) after clozapine dosage had reached $400 \mathrm{mg}$ or the maximum tolerated dose.

\section{Data collection}

Patients were screened 4 - 6 days before the first administration of ECT (those who did not consent to ECT were screened 7 days before clozapine was started); if they met inclusion criteria and consent was obtained, the baseline visit took place during that same visit. All data were collected on data sheets which included all demographic data, information on previous admissions and medication history. A PANSS was performed at baseline (day 0), day 21 and day 42. Clinical case notes were retrospectively accessed after discharge and any documented adverse events, the exact length of stay and the medication data (including concomitant medications) were noted. The investigators were not involved in the primary treatment of any of these patients and had no contact with them other than the study visits.

\section{Statistical analysis}

The data were entered into Excel sheets and data analysis was performed using the Software Package for Social Sciences (SPSS). Chi-square and Student's ttests (Fisher's exact) were utilised depending on the tested variables.

\section{Ethical considerations}

The study was approved by the Committee for Human Research of Stellenbosch University and their prescribed ethical guidelines were strictly adhered to.

\section{Results}

Ten patients were enrolled in the study. Five patients consented to concomitant ECT treatment (EG), whereas the other 5 were started on clozapine only as per the usual treatment protocol (CG). Of these patients 8 were male and 7 reported an educational level of Grade 8 or higher. Nine patients were unemployed and received disability grants. Seven had a diagnosis of schizophrenia and 3 of schizoaffective disorder.

The average number of previous admissions was 3.8 15.2 EG, 2.4 CG; $p=0.245)$. The mean age of onset of illness in the EG was 26.6 years (SD 6.58) and that in the CG 22.8 years (SD $5.805)(p=0.362)$, with mean illness duration in the EG being 8.2 years (SD 5.357) and that in the CG 4.1 years (SD 3.578) $(p=0.198)$.

The average PANSS scores in both groups dropped over the 42-day study period for all three of the subscales measured (i.e. positive, negative, generall. No statistically significant differences could be observed between the two groups at any point for any of the subscales or for the total score. Table I shows a comparison between the two groups' PANSS scores over the course of the study period.

On discharge the average length of stay for both groups in comparison with their most recent admission had decreased, but the difference between the two groups was not statistically significant. With regard to the EG the average calculation was limited to only 4 patients as one patient's most recent previous admission had been in a long-term ward where he had spent almost 7 years. The average clozapine dosage for both groups in comparison with their most recent admission had increased, and once again the difference was not statistically significant. This change amounted to an increase of $20 \mathrm{mg}$ in the EG and an increase of $170 \mathrm{mg}$ in the CG (p=0.352). Table II shows a full comparison of both the average length of stay and clozapine dosage values.

In addition to clozapine, concomitant psychiatric medications were used at discharge in the EG only (with 1 exception) and included valproate (4 cases in EG and 1 in CG), orphenadrine or benzhexol (3 cases), haloperidol (1 case) and sulpiride (1 case). Other concomitant medications during admission comprised

\begin{tabular}{|c|c|c|c|c|c|c|c|c|}
\hline \multirow[b]{2}{*}{ Time point } & \multicolumn{2}{|c|}{ Positive } & \multicolumn{2}{|c|}{ Negative } & \multicolumn{2}{|c|}{ General } & \multicolumn{2}{|c|}{ Total } \\
\hline & EG & $C G$ & EG & CG & EG & CG & EG & CG \\
\hline \multirow{2}{*}{ Day 0} & 29.4 & 30 & 25.8 & 28.6 & 44 & 44.6 & 99.2 & 103.2 \\
\hline & \multicolumn{2}{|c|}{$p=0.85$} & \multicolumn{2}{|c|}{$p=0.287$} & \multicolumn{2}{|c|}{$p=0.215$} & \multicolumn{2}{|c|}{$p=0.508$} \\
\hline \multirow{2}{*}{ Day 21} & 24.8 & 25.8 & 20.6 & 21.6 & 37.6 & 35.2 & 83 & 82.6 \\
\hline & \multicolumn{2}{|c|}{$p=0.188$} & \multicolumn{2}{|c|}{$p=0.386$} & \multicolumn{2}{|c|}{$p=0.144$} & \multicolumn{2}{|c|}{$p=0.305$} \\
\hline \multirow{2}{*}{ Day 42} & 20.8 & 22.2 & 21.8 & 18.6 & 36.4 & 34.8 & 79 & 75.6 \\
\hline & \multicolumn{2}{|c|}{$p=0.84$} & \multicolumn{2}{|c|}{$p=0.64$} & \multicolumn{2}{|c|}{$p=0.093$} & \multicolumn{2}{|c|}{$p=0.36$} \\
\hline
\end{tabular}


Table II. Comparison between average length of stay and clozapine dosages in the two groups

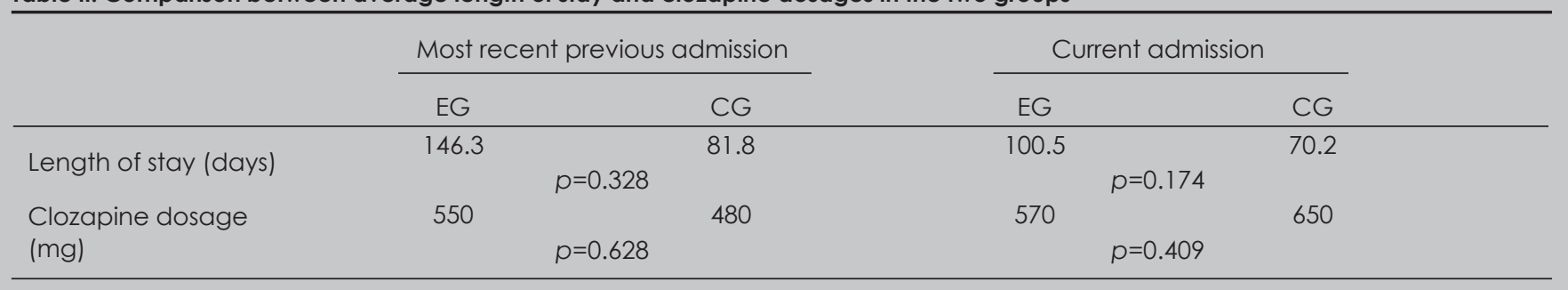

benzodiazepines (7 cases), anti-emetics, promethazine and sorbitol.

Adverse events documented during admission were constipation (1 case), hypersalivation (2 cases), seizures (1 case from the CG and 2 cases from the EG, 1 of which occurred during an ECT session, see below), sedation (1 case) and nausea (1 case). In the EG all but 1 patient had six sessions of ECT. For this patient the last ECT session was cancelled owing to a prolonged seizure during his fifth ECT session which had to be aborted with intravenous diazepam.

\section{Discussion}

The management of patients with schizophrenia has proved to be challenging in various aspects, and especially relevant to this study is the problem of clozapine refractoriness. In this pilot study, ECT-clozapine bitherapy was investigated as one strategy to manage such patients in a resource-constrained setting.

No statistically significant advantage of ECT-clozapine bitherapy above clozapine only could be demonstrated for any of the variables measured in our study setting. However, numerical advantages in terms of decreased length of stay as well as a lower clozapine dose at discharge were observed in the bitherapy group. Although this suggested numerical advantage for the EG in terms of the above variables could probably be attributed to the increased use of concomitant medication in this group, it would be important to note a comparison of the baseline data for the two groups. While not statistically significant, baseline data seemed to suggest that as a group the EG were more severely ill than the CG, as evidenced by (i) higher average length of stay at baseline, and (ii) a higher average clozapine dose on the most recent previous discharge. They also had more previous admissions, but this could just be in keeping with their longer duration of illness.

To date, no prospective control studies have been reported in the literature and the only studies that can possibly be used for comparison are that of Kho et al. ${ }^{18}$ and James and Gray. ${ }^{26}$ However, in James and Gray's prospective open trial on 6 patients with treatment-resistant schizophrenia, patients received six ECT sessions before initiation of clozapine treatment, making comparisons with an ECT-clozapine bitherapy trial difficult. ${ }^{26}$

Kho et al. conducted an open trial with no control group. ${ }^{18} \mathrm{~A}$ few similarities do exist between our methodology and theirs. In both, the study population consisted of a clozapine-refractory schizophrenia group in an inpatient setting. The study groups were both small 111 v. 1015 in the combination group (EG) and 5 in the control group (CG) and the PANSS was used as a primary outcome measure. However, in addition to the absence of a control group, other significant differences in methodology also existed. Kho et al. conducted at least three PANSS assessments before commencing ECT, only starting when the baseline scores remained stable or increased. During this time patients were continued on their clozapine and were adherent for an average of 11.2 weeks before the ECT. PANSS assessments (by two raters) were done weekly and ECT was continued until no further improvement in positive and total PANSS scores were seen, so patients received a mean of 10 ECT sessions during their treatment. Ultimately 8 patients met criteria for remission la drop of at least $30 \%$ from baseline total and positive PANSS scores).

During the course of the study only 1 significant adverse event occurred that could be directly related to the ECT-clozapine biotherapy, in that 1 patient suffered a prolonged seizure during the fifth session of ECT. The seizure was successfully aborted with intravenous diazepam and the patient suffered no further sequelae. To date, no life-threatening side-effects or deaths have been reported in the literature for the combination of ECT and clozapine. ${ }^{27}$ Prescription of ECT-clozapine bitherapy therefore appears to be well tolerated and may be considered reasonably safe with few adverse effects. ${ }^{6}$

Although clozapine blood level measurement could be an important tool in this research setting, it is currently not routinely available in South Africa. It could be helpful in diagnosing clozapine refractoriness, in explaining different responses to treatment and for explaining and monitoring side-effects of clozapine. Future studies of this nature should consider doing clozapine blood level measurement for these reasons. 
The main limitation of this study was the relatively small number of cases and the fact that study staff were not blinded to the treatment. Factors contributing to inability to consent to ECT could also have biased the sample choice. Strengths of the study include the use of a single setting (with established treatment guidelines) as well as a single rater who conducted all assessments.

\section{Conclusion}

This pilot study represents the first controlled trial of ECT-clozapine bitherapy in a population with clozapine-refractory schizophrenia and schizoaffective disorder reported in the literature. In summary, despite the lack of statistically significant results in this study, lessons learnt from strengths and limitations could inform future study designs. The validity of our choice of current clinical practice at Stikland Hospital for the treatment of relapsed, noncompliant, known clozapine-refractory patients with a diagnosis of schizophrenia or schizoaffective disorder was not supported by our results. However, the study did provide us with preliminary evidence for the safety and efficacy of this combination. It would therefore be reasonable to continue to use this strategy in selected cases, at least until other clozapine-refractory treatment strategies become more available in our setting.

The authors would like to acknowledge Karen Cloete's help in preparing a preliminary partial draft of the manuscript.

\section{References}

1. Revicki DA, Luce BR, Weschler JM, Brown RE, Adler MA. Costeffectiveness of clozapine for treatment-resistant schizophrenic patients. Hospital Community Psychiatry 1990; 41: 850-854.

2. Rupp A, Keith SJ. The costs of schizophrenia. Psychiatr Clin North Am 1993; 16, 423

3. Conley RR, Kelly DL. Management of treatment resistance in schizophrenia. Biol Psychiatry 2001; 50: 898-911

4. Chakos M, Lieberman J, Bradford D, Sheitman B. Effectiveness of second-generation antipsychotics in patients with treatment-resistant schizophrenia: a review and metaanalysis of randomised trials. Am J Psychiatry 200 1; 158(4): 51 8-526.
5. Kane J, Honigfeld G, Singer J, Meltzer H and the Clozaril Collaborative Study Group. Clozapine for the treatment-resistant schizophrenic: a double-blind comparison with chlorpromazine. Arch Gen Psychiatry 1988; 45: 789-796.

6. Buckley P, Miller A, Olsen J, Garver D, Miller DD, Csernansky J. When symptoms persist: clozapine augmentation strategies. Schizophr Bull 2001; 27(4): 615-628.

7. Kane JM. Clinical efficacy of clozapine in treatment-refractory schizophrenia: an overview. BrJ Psychiatry 1992; 160: suppl 17, 41-45.

8. Meltzer HY, Burnett S, Bastani B, Ramirez LF. Effects of 6 months of clozapine treatment on the quality of life of chronic schizophrenic patients. Hospital Community Psychiatry 1990; 41: 892-897.

9. Klapheke MM. Follow-up on clozapine and ECT. Convulsive Therapy 1991; 7: 303305

10. al-Semaan Y. Bromocriptine as adjunctive therapy to clozapine in treatment-resistant schizophrenia. Can J Psychiatry 1996; $41:$ 484-485.

11. Buchanan RW, Kirkpatrick B, Bryant N, Ball P, Breier A. Fluoxetine augmentation of clozapine treatment in patients with schizophrenia. Am J Psychiatry 1996: 153: 1625-1627.

12. Carpenter Jr.WT, Breier A, Buchanan RW, Kirkpatrick B, Shepard P, Weiner E. Mazindol treatment of negative symptoms. Neuropsychopharmacology 2000; 23: 365-374

13. Peet M, Horrobin DF. E-E Multicentre Study Group. A dose-ranging exploratory study of the effects of ethyl-eicosapentaenoate in patients with persistent schizophrenic symptoms. J Psychiatr Res 2002; 36: 7-18.

14. Silver $H$, Kushnir $M$, Kaplan A. Fluvoxamine augmentation in clozapine-resistant schizophrenia: an open pilot study. Biol Psychiatry 1996; 40: 671-674.

15. Fink M. Clozapine and electroconvulsive therapy. Arch Gen Psychiatry 1990; 47 290-291

16. Klapheke MM. Clozapine ECT, and schizoaffective disorder, bipolar type. Convulsive Therapy 1991: 7: 36-39

17. Havaki-Kontaxaki BJ, Ferentinos PP, Kontaxakis VP, Paplos KG, Soldatos CR. Concurrent administration of clozapine and electroconvulsive therapy in clozapineresistant schizophrenia. Clin Neuropharmacol 2006; 29(1): 52-56.

18. Kho KH, Blansjaar BA, de Vries S, Babuskova D, Zwinderman AH, Linszen DH. Electroconvulsive therapy for the treatment of clozapine nonresponders suffering from schizophrenia - an open label study. Eur Arch Psychiatry Clin Neurosci 2004; 254(6): 372-379

19. Bloch Y, Pollack M, Mor I. Should the administration of ECT during clozapine therapy be contraindicated? BrJ Psychiatry 1996; 169: 253-254

20. Masiar SJ, Johns CA. ECT following clozapine. BrJ Psychiatry 1991; 158: 133-134.

21. American Psychiatric Association. The Practice of Electroconvulsive Therapy: Recommendations for Treatment, Training and Privileging. A Task Force Report of the American Psychiatric Association. Washington, DC: APA, 1990.

22. Borison RL, Diamond BI. New advances in psychotherapeutic agents: Clozapine. Psychiatr Med 1990; 8: 13-21

23. Baldessarini RJ, Frankenburg FR. Clozapine: a novel antipsychotic agent. N EnglJ Med 1991; 324: 746-754

24. Tranulis C, Mouaffak F, Chouchana L, et al. Somatic augmentation strategies in clozapine resistance - what facts? Clin Neuropharmacol 2006; 29(1): 34-44

25. Kay SR, Fiszbein A, Opler CA. The positive and negative syndrome scale (PANSS) for schizophrenia. Schizophr Bull 1987; 13: 261-276

26. James DV Gray NS. Elective combined electroconvulsive and clozapine therapy. Int Clin Psychopharmacol 1999: 14: 69-72.

27. Braga RJ, Petrides $G$. The combined use of electroconvulsive therapy and antipsychotics in patients with schizophrenia. J ECT 2005; 21 (2): 75-83. 